\title{
Aperiodic Stepwise Growth Model for the Velocity and Orientation Dependence of Solute Trapping
}

\section{Citation}

Aziz, Michael J. and L. M. Goldman. 1987. Aperiodic stepwise growth model for the velocity and orientation dependence of solute trapping. Journal of Materials Research 2(4): 524-527.

\section{Published Version}

http://dx.doi.org/10.1557/JMR.1987.0524

\section{Permanent link}

http://nrs.harvard.edu/urn-3:HUL.InstRepos:2795824

\section{Terms of Use}

This article was downloaded from Harvard University's DASH repository, and is made available under the terms and conditions applicable to Other Posted Material, as set forth at http:// nrs.harvard.edu/urn-3:HUL.InstRepos:dash.current.terms-of-use\#LAA

\section{Share Your Story}

The Harvard community has made this article openly available.

Please share how this access benefits you. Submit a story.

Accessibility 


\title{
Aperiodic stepwise growth model for the velocity and orientation dependence of solute trapping
}

\author{
L. M. Goldman \\ Division of Applied Sciences, Hanuard University, Cambridge, Massachusetts 02138 \\ M. J. Aziz") \\ Solid State Division, Oak Ridge National Laboratory, Oak Ridge, Tennessee 37831
}

(Received 30 January 1987; accepted 8 April 1987)

\begin{abstract}
An atomistic model for the dependence on interface orientation and velocity $v$ of the solute partition coefficient $k$ during rapid solidification is developed in detail. Starting with a simple stepwise growth model, the simple continuous growth model result is obtained for $k(v)$ when the growth steps are assumed to pass at random intervals rather than periodically. The model is applied to rapid solidification of silicon. Crystal growth at all orientations is assumed to occur by the rapid lateral passage of (111) steps at speeds determined by the interface velocity and orientation. Solute escape is parametrized by a diffusion coefficient at the edge of the moving step and a diffusion coefficient at the terrace, far from the step edge. The model results in an excellent fit to data for the velocity and orientation dependence of $k$ of $\mathrm{Bi}$ in $\mathrm{Si}$.
\end{abstract}

\section{INTRODUCTION}

When solidification of binary liquid alloys occurs at interface velocities $v$ on the order of or greater than the diffusive velocity of the solute atoms at the liquid/solid interface, nonequilibrium fractions $k$ of the solute in the liquid at the interface are incorporated into the solid. This phenomenon is known as solute trapping. On the one hand, the continuous growth model ${ }^{1}$ (CGM) fits the $k(v)$ data $^{2}$ quite well. In this model, solute-solvent redistribution across the interface and the simultaneous advance of the interface are treated as separate processes that occur under microscopically steady-state conditions. As originally derived, however, the CGM does not provide a detailed atomistic description of the process of solute trapping. On the other hand, the stepwise growth model ${ }^{3}$ (SGM) provides a detailed atomistic picture of the solute trapping process: crystallization of a monolayer, occuring by the rapid lateral passage of a growth step, and solute-solvent redistribution alternate in time. However, in its simplest form the SGM yields a form for $k(v)$ with too strong a velocity dependence to fit the data.

Using pulsed laser melting techniques, Aziz and White ${ }^{4}$ recently measured the orientation dependence of $k$ at constant $v$ and accounted for their results with an extension of the CGM. They assumed, as did Pfeiffer, that growth occurs by the periodic rapid lateral passage of $\{111\}$ steps separated by an interval determined by the velocity and orientation of the interface, as shown in Fig. 1. They also assumed that all of the solute escape occurs at the edge of the moving step and that there is no escape once the solute atom is incorporated into the ter-

\footnotetext{
- Permanent address: Division of Applied Sciences, Harvard University, Cambridge, Massachusetts 02138.
}

race. Their model recovers the CGM velocity dependence and accounts fairly well for the observed sharp rise in $k$ as the $\{111\}$ orientation is approached. However, the model predicts that $k \rightarrow 1$ at $\{111\}$ where the ledge velocity diverges. The data indicate that, while significantly greater than at nearby orientations, $k$ at $\{111\}$ is not unity but rather is in the range $0.2-0.5$, depending on velocity. Apparently there is a need to account for escape from the terrace.

In this article we first show that the simple CGM form for $k(v)$ results from the lateral passage of steps if the interval between the passage of steps is assumed to be random, rather than constant. We then combine this model for the escape of solute that has been incorporated into the terrace with the model of Aziz and White for solute incorporation at the step edge. We obtain excellent agreement with the data for velocity and orientation dependence of solute partitioning of $\mathrm{Bi}$ in $\mathrm{Si}$.

\section{THE MODEL}

\section{A. Equivalence of random interval SGM and simple CGM}

In the SGM solute trapping is a two-stage process. The first stage is the incorporation of a monolayer of

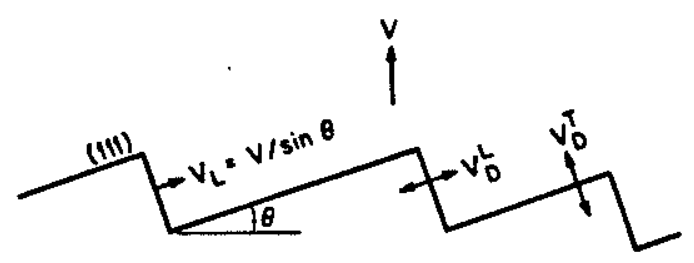

FIG. 1. Solute trapping by the lateral passage of random length (111) steps, with solute escape through the step edge and step terrace. 
height $\lambda$ in the liquid at the interface into the solid without a change in composition. In this process solute atoms are forced onto lattice sites when their solvent neighbors assume lattice positions. The solute atoms may experience an increase in their chemical potential during this process. This stage is assumed to be very fast, as would be the case if it occurred by the rapid lateral passage of a growth step. The second stage is the diffusion of solute back into the liquid. Diffusion comes to an end when the next monolayer is added to the crystal, permanently trapping the remaining solute atoms in the solid. The layers are added periodically, with period $\tau=\lambda / v$, where $\lambda$ is the monolayer step height. In the interval between the addition of monolayers the decay of $C_{s i}$, the composition of the solid interface layer is given by

$$
\frac{d C_{s i}}{d t}=-\left(\frac{D_{i}}{\lambda^{2}}\right)\left[C_{s i}(t)-k_{e}\left(T_{i}\right) C_{l i}\right],
$$

where $C_{l i}$ is the composition of the liquid at the interface, assumed to be constant, $D_{i}$ is the coefficient for interdiffusion across the interface, and $k_{e}\left(T_{i}\right)$ is the equilibrium partition coefficient (i.e., the ratio of the solidus composition to the liquidus composition) at the interface temperature $T_{i}$. Integrating this expression, defining the reduced concentration $C_{r}(t)=C_{s i}(t) / C_{l i}$, and applying the initial condition that $C_{r}(0)=1$ yields

$$
C_{r}(t)=\left[k_{e}+\left(1-k_{e}\right) \exp -\left(D_{i} t / \lambda^{2}\right)\right] .
$$

Note that $C_{r}(t)$ is not the partition coefficient, but is rather the instantaneous composition of the solid interface layer relative to $C_{l i}$. The final value of $C_{r}(t)$ occurs when addition of the next monolayer at time $t=\tau$ brings diffusion to a halt. If the diffusive speed is defined as $v_{D}=D_{i} / \lambda$ and the normalized velocity as $\beta=v / v_{D}$, we have the $S G M$ result:

$$
k=C_{r}(\tau)=k_{e}+\left(1-k_{e}\right) \exp -(1 / \beta) .
$$

If, instead, we assume that the layers are added randomly in time, rather than periodically, then the probability that a given layer is not covered at time $t$, but is then covered in the next time interval $d t$ is given by

$$
P(t) d t=\frac{e^{-t / \tau} d t}{\tau} .
$$

The composition of the solid, averaged over many $\tau$, is then given by

$$
k=\int_{0}^{\infty} C_{r}(t) P(t) d t=\frac{\left(\beta+k_{e}\right)}{(\beta+1)},
$$

which is the simple CGM result. It should be noted that in the case of the SGM it is not necessary to add complete atomic layers (i.e., a whole layer at a time). In fact, since in this model the action at the interface is independent of any lateral correlation, the layers can be added in any fashion that is periodic with period $\tau$. However, there are few ways of adding layers that satisfy periodicity and are physically plausible. One way that satisfies both conditions is the lateral passage of growth steps.

\section{B. Application to redistribution at step edges and at terraces}

We now apply this result to the orientation dependence experiment. It was originally suggested that trapping occurs only at the step edges moving with lateral speed $v_{L}=v / \sin \theta$, where $\theta$ is the inclination angle from (111), and that no escape flux passes through the terrace. The present model allows for an additional escape flux through the terraces. We introduce a second parameter $v_{D}^{T}$, the speed of diffusion out of the terrace once the solute atom is incorporated into the solid monolayer. Since in this position the solute atom is triply or quadruply coordinated, we expect $v_{D}^{T}<v_{D}^{L}$, the latter being the corresponding quantity at the step edge where the atom is doubly coordinated.

We retain the original assumption that trapping occurs at the step edges and is characterized by the CGM, i.e., the amount of solute initially incorporated into the terrace by the lateral passage of the step is given by Eq. (2) of Ref. 4:

$$
C_{r}(0)=\frac{\left\{\left[v_{L}(\theta) / v_{D}^{L}\right]+k_{e}\right\}}{\left\{\left[v_{L}(\theta) / v_{D}^{L}\right]+1\right\}} .
$$

In this picture the kinetics of continuous lateral ledge motion versus lateral solute-solvent redistribution lead to a $C_{r}(t=0)$ representative of partial trapping of solute onto the terrace. This initial trapping process is described by the simple CGM, except that $v_{L}$ replaces $v$ and $v_{D}^{L}$ replaces $v_{D}$. (Of course, the process could be equally well described by the aperiodic lateral passage of kinks.)

Some solute atoms then escape through the terrace before the next ledge passes and permanently covers the remaining solute. This process is accounted for by integrating Eq. (1), using the initial condition given by Eq. (6). The result is very similar to Eq. (2):

$$
\begin{aligned}
C_{r}(t)= & k_{e}+\left\{\left[\left(\beta_{l}+k_{e}\right) /\left(\beta_{l}+1\right)\right]-k_{e}\right\} \\
& \times \exp -\left(D_{i}^{T} t / \lambda^{2}\right),
\end{aligned}
$$

where $D_{i}^{T}$ is the diffusion coefficient perpendicular to the terrace and $\beta_{l}=v_{L} / v_{D}^{L}$. If the ledges are randomly spaced (as in Fig. 1), then the measured partition coeffcient is an average over all terrace lengths. This is formally identical to having layers added with random period, and we can therefore apply Eq. (5) to obtain the final partition coefficient:

$$
k=\frac{\left\{k_{e}+\beta_{t}\left[\left(\beta_{l}+k_{e}\right) /\left(\beta_{1}+1\right)\right]\right\}}{\left(\beta_{t}+1\right)},
$$

where $\beta_{i}=(v / \cos \theta) / v_{D}^{T}$. Expression (8) will be re- 
ferred to as the aperiodic stepwise growth model (ASGM), as it describes solute trapping as occurring by the aperiodic passage of steps. Note that if $v_{D}^{T}=0$ the above expression reduces to that of Aziz and White for no escape through the terraces.

\section{COMPARISON OF THEORY TO DATA}

The two fitting parameters in the ASGM are $v_{D}^{T}$ and $v_{D}^{L}$. In Fig. 2 we compare the ASGM to the data for the orientation dependence of $k$ at constant velocity. Two distinct velocities were achieved experimentally, 1.7 and $5 \mathrm{~m} / \mathrm{s}$. For the curves drawn in Fig. 2, the fitting parameters were $v_{D}^{T}=6 \mathrm{~m} / \mathrm{s}$ and $v_{D}^{L}=20 \mathrm{~m} / \mathrm{s}$. The former is determined solely by fitting $k$ at (111). The latter is then varied to bring the curves into agreement with the rest of the data. The resulting fit is excellent. In Fig. 3 we compare the ASGM to the data for velocity dependence at $(001)$. While the fit of the ASGM to the data is acceptable, the simple CGM provides a somewhat better fit to the velocity-dependence data. Heat flow calculations were used to determine the interface velocity in the orjentation-dependence experiment. In the velocity-dependence experiment $v$ was measured directly. The values of the fitting parameters $v_{D}^{T}$ and $v_{D}^{L}$ were determined by a fit to the former data. The discrepancy between the latter data and the ASGM may arise from small systematic errors in the heat flow calculations of $v$ and their effect on the fitting of $v_{D}^{T}$ and $v_{D}^{L}$. (Note that the two circles in

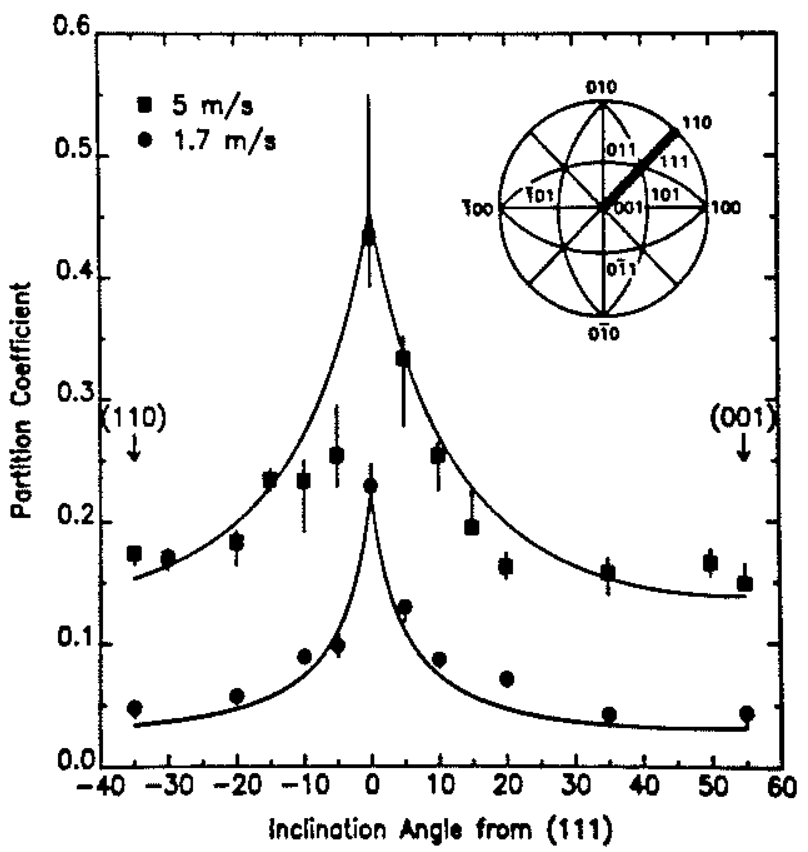

FIG. 2. Comparison of aperiodic stepwise growth model to data for the orientation dependence of partition coefficient at $1.7 \mathrm{~m} / \mathrm{s}$ (circles) and $5 \mathrm{~m} / \mathrm{s}$ (squares). The fitting parameters in the ASGM are $v_{D}^{T}=6 \mathrm{~m} / \mathrm{s}$ and $v_{D}^{L}=20 \mathrm{~m} / \mathrm{s}$.

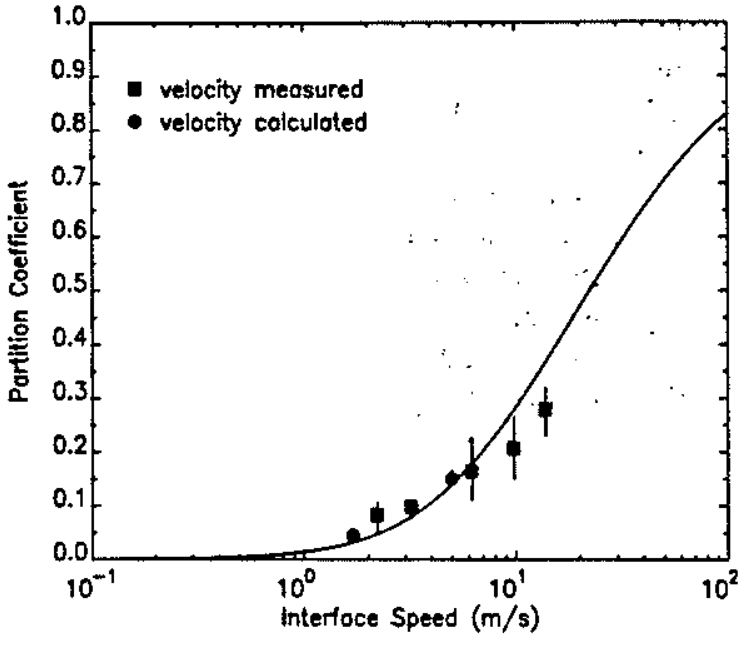

FIG. 3. Comparison of aperiodic stepwise growth model to data for the velocity dependence of partition coefficent at the $(001)$ orientation. The fitting parameters are $v_{D}^{T}=6 \mathrm{~m} / \mathrm{s}$ and $v_{D}^{L}=20 \mathrm{~m} / \mathrm{s}$.

Fig. 2, which came from the orientation-dependence experiment, show better agreement with the ASGM than do the rest of the data.)

Consider the height of the ledges in Fig. 1. If the ledge is more than one atomic layer in height then the solute composition of the buried layers (which are never free to exchange solute atoms with the liquid through the terraces since the motion of a fourfold-coordinated dopant would be governed by the bulk diffusivity, which is exceedingly small) should be described by the model of Aziz and White. The overall partition coefficient would be the average of the partition coefficient of all of the layers. If the ledges were at least two atomic layers high, then the $k$ of the top layer is described by the ASGM, while that of the remaining layer is described by the Aziz-White model. Since at (111) $v_{L}$ diverges and $k$ of the bottom layer will always be 1 in the Aziz-White model, the minimum overall $k$ that could be measured at any speed would be 0.5 . The data in Fig. 2 show $k=0.23$ at $v=1.7 \mathrm{~m} / \mathrm{s}$ for (111). Therefore, within the context of this model, ledges must be only one atomic layer in height. This simple argument holds only at or near (111). However, quantitative analysis of our model, including the possibility of large step heights at orientations far from (111) and both forms of solute escape, indicates that the ASGM is consistent with the data only if growth at any orientation occurs by the lateral passage of monolayer $\{111\}$ steps. This argument does not rule out the possibility of large facets advancing by the nucleation at the reentrant comers and subsequent motion of monolayer growth steps.

In the well-known "facet effect," 6 at a (111) facet appears to be higher than $k$ at an unfaceted orientation during Czochralski growth. This is not inconsistent 
with ASGM if the effect is relatively small. Using $v_{D}^{T}$ and $v_{D}^{t}$ obtained from high $v$ data, we estimate that at these speeds differences of $\sim 1 \%$ might be found for $\mathrm{Bi}$ in $\mathrm{Si}$. It is conceivable that for other dopants there might be a somewhat more pronounced facet effect. However, an effect such as that reported for Te in InSb, ${ }^{7}$ in which $k$ at (111) is greater than unity despite $k$ at other orientations being less than unity, is inconsistent with simple models such as the ASGM. More complicated trapping models that include adsorption at the interface $e^{8-12}$ are necessary to explain such behavior.

\section{CONCLUSIONS}

We have developed a simple atomistic model for solute trapping that is based on (a) random intervals between the passage of growth steps and (b) two chances for solute escape: one at the edge of the moving step and another out of the terrace. With one fitting parameter to characterize each flux, the model yields an excellent fit to the data for the velocity and orientation dependence of solute trapping of $\mathrm{Bi}$ in $\mathrm{Si}$.

\section{ACKNOWLEDGMENTS}

We thank D. M. Zehner, J. Y. Tsao, S. Axelrod, W. J. Boettinger, and J. H. Perepezko for helpful discussions. One of us (M.J.A.) was supported in part by an appointment to the United States Department of Ener- gy Faculty Research Participation Program administered by Oak Ridge Associated Universities. Work at Harvard was supported by the Office of Naval Research under Contract No. N00014-85-K-0023 and by the Harvard Materials Research Laboratory under National Science Foundation Contract No. DMR-83-16979.

\section{REFERENCES}

'M. J. Aziz, Appl. Phys. Lett. 43, 552 (1983).

${ }^{2}$ M. J. Aziz, J. Y. Tsao, M. O. Thompson, P. S. Peercy, and C. W. White, Phys. Rev. Lett. 56, 2489 (1986).

${ }^{3}$ M. J. Aziz, J. Appl. Phys. 53, 1158 (1979).

4M. J. Aziz and C. W. White, Phys. Rev. Lett. 57, 2675 (1986).

${ }^{5}$ H. Pfeiffer, J. Cryst. Growth 52, 350 (1981).

'M. G. Mil'vidskii and A. V. Berkova, Sov. Phys. Solid State 5, 374 (1963).

'J. B. Mullin and K. F. Hulme, J. Phys. Chem. Sol. 17, 1 (1960).

R. N. Hall, J. Phys. Chem. 57, 836 (1953).

${ }^{9}$ A. A. Chernov, in Growth of Cnystals, edited by A. V. Shubnikov and N. N. Sheftal (Consultants Bureau, New York, 1962), Vol. 3, p. 35.

${ }^{10} \mathrm{~J}$. C. Brice, The Growth of Crystals from the Melt (North-Holland, Amsterdam, 1965), pp. 63-69.

"J. W. Cahn, S. R. Coriell, and W. J. Boettinger, in Laser and Electron Beam Pracessing of Materials, edited by C. W. White and P. S. Peercy (Academic, New York, 1980), pp. 89-103.

${ }^{12}$ M. J. Aziz, Ph. D. thesis, Harvard University, 1983, pp. 76-82. 